\title{
AVALIAÇÃO DO COMPORTAMENTO MECÂNICO DO POLIÉSTER ORTOFTÁLICO RETICULADO COM ADIÇÃO DE PET RECICLADO
}

\author{
Sandra Cunha Gonçalves, Celso Carlino Fornari
}

\section{RESUMO}

Um dos grandes problemas enfrentados pela sociedade moderna está em encontrar uma destinação final adequada para o excesso resíduo sólido gerado, principalmente de produtos descartáveis. Neste contexto, a utilização do PET reciclado, como reforço em compósitos, é uma idéia que surge com o intuito de buscar alternativas para o reaproveitamento deste resíduo. Neste trabalho, estudou-se a influência da variação do PET nas propriedades mecânicas de compósitos de matriz poliéster. Foram confeccionados corpos de prova de matriz pura e com 5 e $10 \%$ de PET nas granulometrias de 40,60 e 80 mesh. Avaliou-se o comportamento mecânico dos compósitos por meio de testes de flexão em três pontos. Os resultados mostraram um aumento gradual no módulo de elasticidade com o aumento do percentual de PET nas menores granulometrias, resultando na redução significativa da deformação em relação à matriz.

Palavras-chave: Avaliação mecânica, PET reciclado, Compósito polimérico.

\begin{abstract}
One of the major problems faced by modern society is to find a suitable disposal for excess solid waste generated, particularly disposable products. In this context, the use of recycled PET as reinforcement in composites, is an idea that arises in order to seek alternatives for the recycling of this waste. We studied the influence of the variation in the mechanical properties of PET fiber reinforced polyester. Test specimens were made of pure matrix and 5 to $10 \%$ of PET in the particle size of 40,60 and 80 mesh. We evaluated the mechanical behavior of composites by means of bending tests on three points. The results showed a gradual increase in modulus with increasing percentage of PET in smaller particle sizes, resulting in significantly reducing deformation in relation to the matrix.
\end{abstract}

Keywords: mechanical evaluation, PET recycled, polymer composite.

INTRODUÇÃO

Material compósito pode ser definido como um material formado por uma mistura ou combinação de dois ou mais constituintes que diferem na forma e na composição química, e que, na sua essência, são insolúveis e miscíveis entre si. Sua importância em engenharia vem do fato de que, ao combinar-se dois ou mais materiais diferentes, obtém-se um material compósito cujas propriedades são a soma das contribuições de cada uma das propriedades dos componentes (AHMAD et al, 2007). Com isto, vemos que a tecnologia tem trazido grandes benefícios à 
sociedade, proporcionando seu desenvolvimento. Entretanto, existem também os efeitos negativos, proporcionando problemas ecológicos e ambientais. Com o aumento significativo da produção e do consumo de produtos industrializados, o reaproveitamento de resíduos surge como uma das mais importantes atividades de controle ambiental, agregando valores econômicos e desenvolvimento tecnológico.

Neste ínterim, o desenvolvimento de pesquisas que contemplem a reutilização de resíduos é de grande relevância, dentro de uma visão que trata estes poluentes como matérias-primas importantes para aplicação de compósitos. Segundo Marconcini (2008), a reciclagem do PET poli(tereftalato de etileno) representa um dos exemplos mais importantes de sucesso da reciclagem de polímeros, sendo que um dos principais mecanismos responsáveis pelo aumento da reciclagem pós-consumo do PET é a grande variedade de aplicações para os reciclados. O Poli Tereftalato de Etileno (PET) é um plástico de engenharia que apresenta alta resistência á degradação, tanto atmosférica quanto biológica (AHMAD et al, 2007). Desta maneira, muitos trabalhos têm sido desenvolvidos de forma a buscar alternativas viáveis para a reutilização ou reciclagem do PET (ZANIM; BEZERRA; MANCINI, 1998). A tecnologia "bottle-to-bottle" reutiliza PET reciclado para reprocessar novas embalagens para alimentos, onde na Europa e Estados Unidos é permitido. O reprocessamento mecânico tem etapas variáveis para o processo, o que depender do tipo de polímero. Geralmente ocorre a etapa de separação seguida de moagem, lavagem e a etapa de secagem. $O$ processo químico de reciclagem envolve a transformação química do polímero. $O$ polímero diminui o seu peso molecular por meio de despolimerização ou mesmo por diminuição do comprimento da cadeia (ruptura) (EHRIG; CURRY, 1992). São utilizadas reações químicas de hidrólise ou alcoólise, decomposição térmica (aquecimento na ausência de oxigênio, gaseificação ou hidrogenização) e decomposição térmica acompanhada de catalisador (OLIVEIRA; FILHO, 2009).

A justificativa para a utilização do PET como carga em materiais compósitos é uma proposta que surge como mais uma alternativa para o reaproveitamento de um material de difícil decomposição, além do desenvolvimento de materiais com melhores propriedades. $\mathrm{O}$ objetivo principal deste trabalho foi avaliar o comportamento mecânico, através do ensaio de flexão em três pontos, da adição do PET em frações de $5 \%$ e $10 \%$ como carga em matriz polimérica a base de poliéster, comparando-o com a matriz pura, para permitir o estudo do potencial de utilização deste material.

\section{METODOLOGIA}

- MATERIAIS

- Resina Poliéster;

- Estireno;

- Álcool;

- Cobalto;

- peróxido orgânico líquido, denominado de MEK (metil etil cetona);

- Carga - Poli Tereftalato de Etileno (PET) reciclado - PETr. 
- MÉTOdOS

O procedimento de fabricação dos corpos de prova constituiu-se em:

- As garrafas PET foram inicialmente cortadas e moídas em moedor manual e posteriormente moídas em moinho de facas. As fibras moídas foram selecionadas quanto a sua granulometria, seguindo a norma ABNT NBR 10439/1988 - métodos A. Para este ensaio foi utilizado um agitador de peneiras marca BERTEL com jogo de peneiras de 40 mesh, 60 mesh, 80 mesh e fundo cego.

- Foi utilizada resina poliéster insaturada ortoftálica pré-acelerada com sal de cobalto e iniciador de reticulação metil etil cetona em solução de estireno. Todo o processo de pesagem foi realizado em balança de precisão.

- Na produção do compósito utilizou-se $5 \%$ e $10 \%$, em relação a quantidade de resina, do PET moído nas granulometrias de 40, 60 e 80 mesh.

- Após a produção os Corpos de prova foram submetidos inicialmente a cura em temperatura ambiente por 60 minutos e após este período, foram levados a estufa, préaquecida em $80^{\circ} \mathrm{C}$, por 60 minutos. Os ensaios de flexão foram conduzidos em equipamento de ensaio universal com deslocamento negativo obedecendo os padrões normativos.

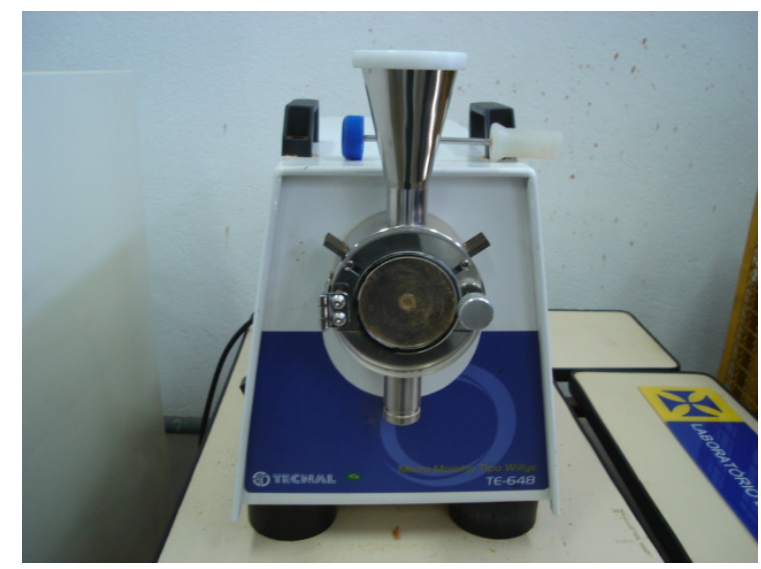

Figura 1: Moinho de facas utilizado para moer o PETr. 


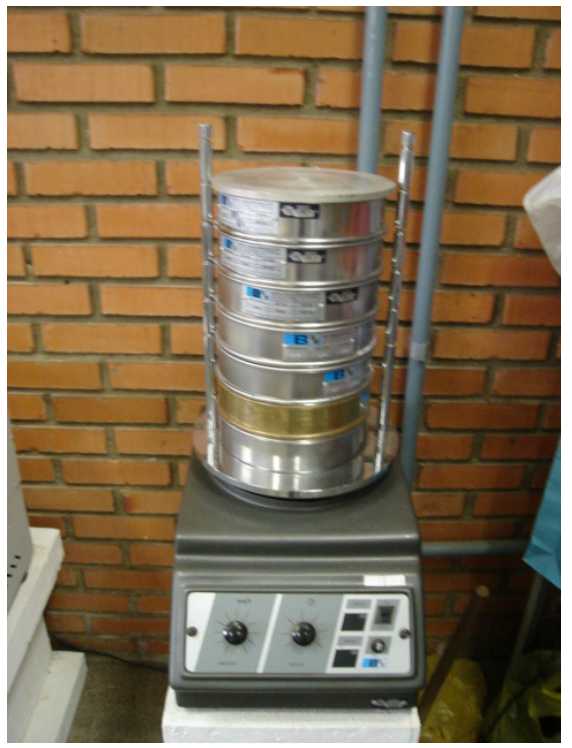

Figura 2: Conjunto de peneiras e agitador usado para separar o material moído.

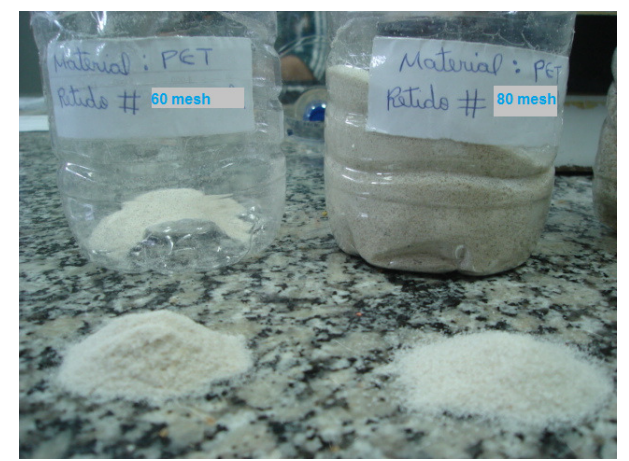

Figura 3: material PETr moído e separado de acordo com a granulometria.

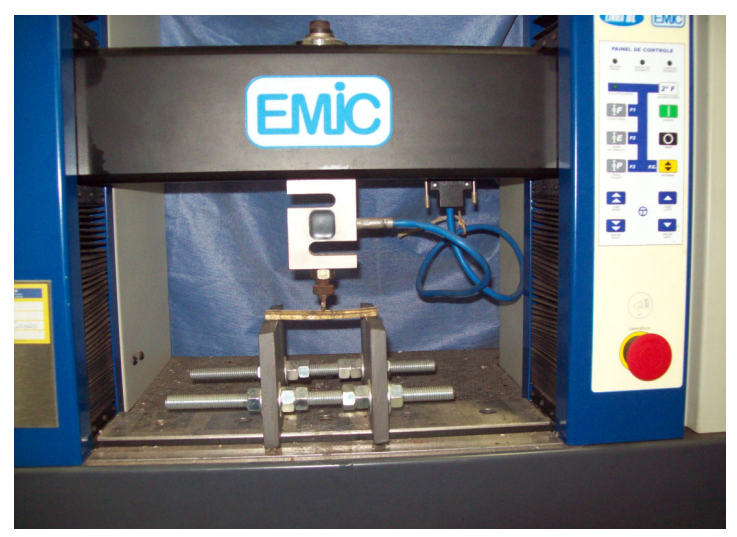

Figura 4: Configuração do ensaio de flexão em três pontos. 


\section{RESULTADOS E DISCUSSÃO}

Através dos resultados obtidos com os ensaios de flexão em três pontos foi possível obter as informações descritas a seguir, sabendo que a estrutura do compósito varia em função dos seus constituintes e estes são os responsáveis pelas propriedades mecânicas do material, onde cada constituinte contribui no somatório geral das propriedades do compósito (AHMAD et al, 2007). A figura 5 apresenta os valores médios da tensão na flexão, para a matriz e os compósitos moldados com 5 e $10 \%$ nas granulometrias de 40,60 e 80 mesh.

A maior tensão observada na figura 5 ocorreu para a matriz, este comportamento pode ser explicado porque a incorporação de carga ao compósito pode gerar uma zona de fragilidade, pois no processo de reticulação do poliéster, ocorre a união das macromoléculas por meio de ligações químicas permanentes. Neste processo a interferência de partículas estranhas ao polímero pode causar modificações em relação ao comportamento mecânico do compósito. A fração volumétrica específica de cada partícula exige que as cadeias moleculares se acomodem de maneira forçada em torno da partícula de PET.

Desta forma, a partícula cria um espaço vazio de polímeros, do tipo poliéster, em torno de si mesma, gerando um ponto heterogêneo no compósito, isto é, uma espécie de ilha cercada por poliéster reticulado. Pode-se explicar este fato levando em consideração o fato de que partículas dispersas no seio polimérico podem agir como pontos heterogêneos que se localizam entre as moléculas adjacentes, dificultando a dissipação de energia transmitida ao material. Sendo assim, pode-se dizer que as partículas maiores de PET, quando presentes na matriz do compósito, podem agir de forma a inibir a distribuição de energia transmitida ao sistema, dificultando mais a aproximação das moléculas do polímero ao passo que partículas menores interferem de forma mais amena.

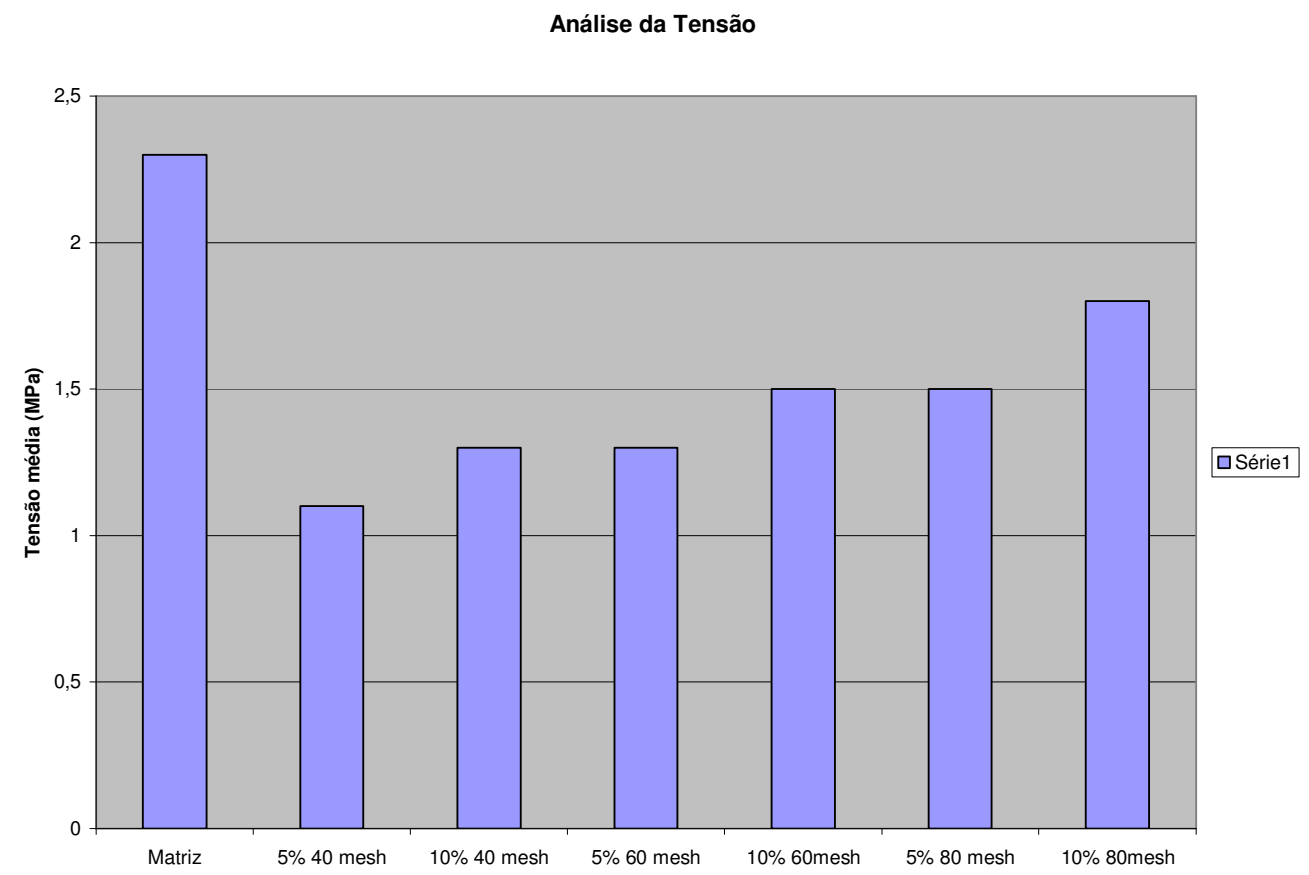

Figura 5: Gráfico comparativo entre a tensão média dos corpos de prova com matriz pura, 5\% e $10 \%$ de PET em granulometrias diferenciadas. 
Entretanto, as pequenas partículas apresentam maior área de contato relativa, o que favorece um melhor acoplamento ou ligação com o próprio polímero. Pudemos observar este fato analisando os resultados obtidos, que foram significativos, logo, pode-se dizer que o tamanho das partículas é um fator importante nas propriedades mecânicas do material compósito.

$A$ adição de PET levou a um decréscimo em todos os compósitos em relação a matriz, entretanto, a medida em que se reduziu as partículas e se aumentou o percentual de carga foi possível observar um acréscimo na tensão. Conforme mostra a Tabela 1.

Tabela 1: Percentual de acréscimo da tensão em função da granulometria e variação volumétrica.

\begin{tabular}{c|c|c}
\hline $\begin{array}{c}\text { Relação entre } \\
\text { Granulometrias }\end{array}$ & $\begin{array}{c}\text { Percentual do acréscimo da tensão para } \\
\text { compósitos com 5\% de PET }\end{array}$ & $\begin{array}{c}\text { Percentual do acréscimo da tensão } \\
\text { para compósitos com 10\% de PET }\end{array}$ \\
\hline 60 mesh - 40 mesh & $18 \%$ & $15 \%$ \\
\hline 80 mesh - 60 mesh & $15 \%$ & $20 \%$ \\
\hline 80 mesh -40 mesh & $36 \%$ & $38 \%$ \\
\hline
\end{tabular}

A figura 6 apresenta os valores do módulo de elasticidade, para a matriz e os compósitos moldados com 5 e $10 \%$ nas granulometrias de 40,60 e 80 mesh. A análise dos resultados mostrou um acréscimo gradual bastante significativo, em relação a matriz, de todos os compósitos, conforme mostram os resultados apresentados na tabela 2 .

Tabela 2: Percentual de acréscimo do módulo de elasticidade em função da granulometria e variação volumétrica.

\begin{tabular}{c|c|c}
\hline Relação entre compósitos & $\begin{array}{c}\text { Percentual de acréscimo para } \\
\text { compósitos com 5\% de PET }\end{array}$ & $\begin{array}{c}\text { Percentual de acréscimo para compósitos } \\
\text { com 10\% de PET }\end{array}$ \\
\hline 40 mesh - matriz & $78 \%$ & $155 \%$ \\
\hline 60 mesh - matriz & $105 \%$ & $204 \%$ \\
\hline 80 mesh - matriz & $156 \%$ & $19 \%$ \\
\hline 60 mesh -40 mesh & $15 \%$ & $15 \%$ \\
\hline 80 mesh -60 mesh & $25 \%$ & $37 \%$ \\
\hline 80 mesh -40 mesh & $44 \%$ & $249 \%$ \\
\hline
\end{tabular}

O módulo de elasticidade está relacionado com a energia de ligação e o fator de empacotamento do arranjo atômico, que neste trabalho foi determinado através da análise da tensão - deformação obtida no ensaio de tração na flexão. Este módulo avalia a resistência do material a deformação elástica, sendo então uma medida da sua rigidez, na maioria das aplicações da engenharia não se deseja a ocorrência de deflexões, busca-se materiais com alto módulo de elasticidade. 


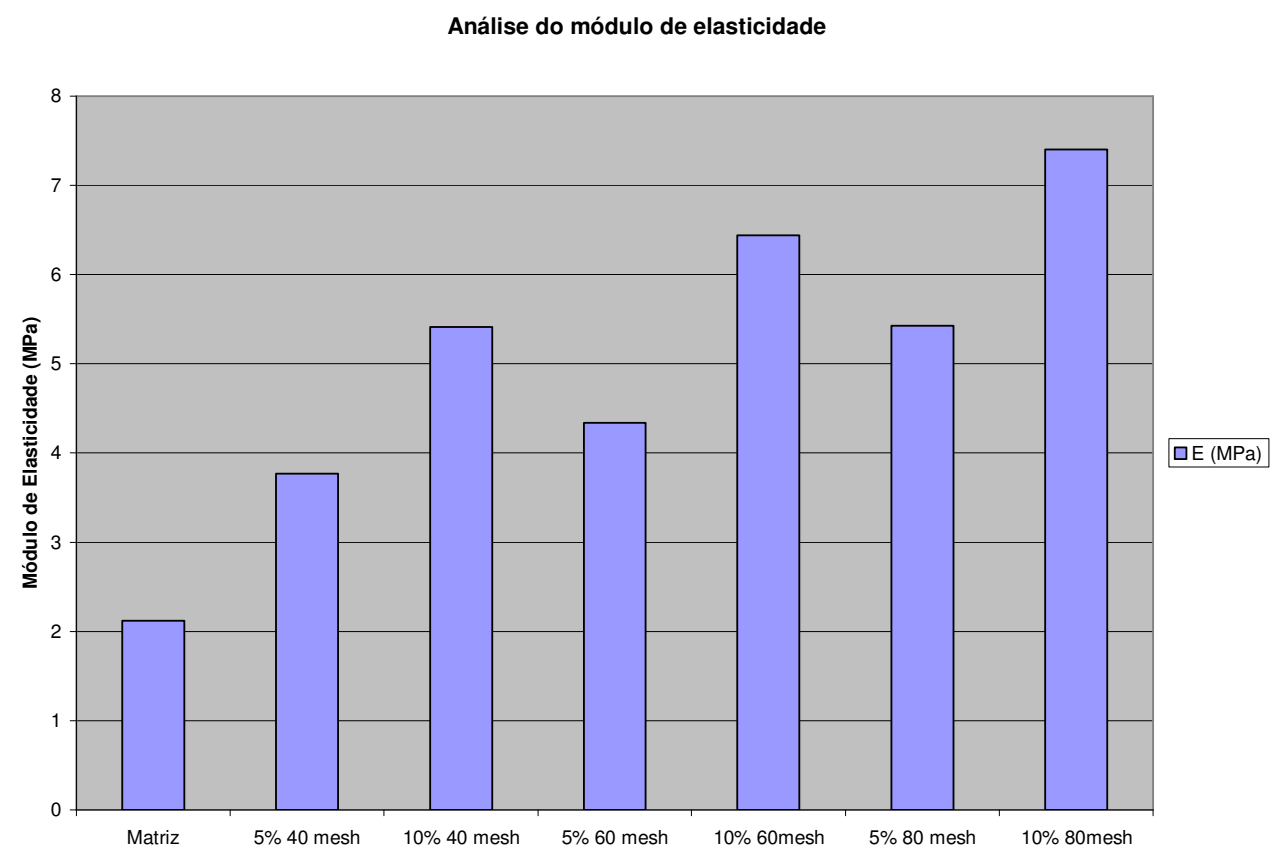

Figura 6: Módulo de elasticidade para cada percentual de PET em granulometrias diferenciadas.

\section{CONCLUSÃO}

Os resultados mostraram que há uma viabilidade técnica para a utilização dos compósitos produzidos com matriz de resina poliéster ortoftálica com adição de PET reciclado, principalmente se o objetivo for empregar um material mais rígido. Foi possível observar também que os resultados foram melhores para granulometrias menores, indicando que há uma influencia nos tamanhos das partículas.

\section{REFERÊNCIAS}

ABNT: Associação Brasileira de Normas Técnicas. NBR 10439: Plástico - Determinação do tamanho de partículas - Métodos das peneiras - Método de ensaio. Rio de Janeiro, 1988.

AHMAD, I.; RAML, A.; MOKHILAS, S.N.; BAKAR, D.R.A. Dirrect Usage of Products of Poly(ethylene terephthalate) glycolysis for manufaturing of rice husk/unsaturated polyester composite. Iranian Polymer Journal, v.16, n4, p.233-239. 2007.

CALLISTER, W.D.Jr. Ciência e Engenharia de Materiais: Uma Introdução. Rio de Janeiro, LTC, 2002. 
CARVALHO, L.H.; CAVALCANTI, W.S. Propriedades mecânicas de tração de compósitos poliéster / tecidos híbridos sisal/ vidro. Artigo técnico. Polímeros: Ciência e Tecnologia, vol. 16, no 1, p. 33-37, 2006.

DE PAOLI M.A.; SPINACÉ M.A.S.; ROMÃO W. Poly (ethylene terephthalate), PET: a review on the synthesis processes, degradation mechanisms and its recycling. Polímeros, v 19, n2, p. 121, 132, 2009.

EHRIG R.J.; CURRY M.J. Plastics recycling: products and processs. Ehrig R.J. Oxford University Press: New York, 1992.

GORNI, A.A. A evolução dos materiais poliméricos ao longo do tempo. Revista plástico industrial, 2003. Disponível em: <http://www.gorni.eng.br/hist_pol.html> acesso em 21/02/2010.

MARCONCINI J.M. Comportamento Interfacial de Compósitos de Poli (Tereftalato de Etileno) Reciclado (PETr) com Fibras de Bagaço de Cana. Comunicado Técnico. EMBRAPA. 2008

MATTOSO, L.H.C. Comportamento interfacial de compósitos de Poli (tereftalato de Etileno) Reciclado (PETr) com fibra de bagaço de cana. Comunicado Técnico. São Carlos - SP, 2008.

OLIVEIRA P.T.S.; FILHO G.E.C.P. Levantamento da situação da reciclagem de materiais plásticos no município de Campo Grande MS. IV Encontro Nacional e II Encontro Latino Americano sobre Edificações e Comunidades Sistentáveis. 2009.

SOBRINHO, L.L. Desenvolvimento de matriz polimérica para material compósito visando o reforço de dutos de aço.2005. Tese de Doutorado, UFRJ, Rio de Janeiro-RJ.

SILVA, V.P.. Compósitos Poliméricos de fibras curtas de sílica / silicona. 2005. Dissertação de mestrado, Unicamp, Campinas.

TEODÓSIO A.S.S.; FRANCELINO S.L.G.D. Estruturas da cadeia reversa: "caminhos" e "descaminhos" da embalagem PET. Produção, v.16, n 3, p 429-441, set/dec. 2006.

ZANIM M., BEZERRA M.N., MANCINI S.D. Reciclagem de PET advindo de garrafas de refrigerante pósconsumidos. Polímeros: Ciência e Tecnologia. P. 68-75, abri-jun, 1998. 